\title{
A Cascade Linear Active Disturbance Rejection Controller for Vector Control System of PMSM
}

\author{
Hourao Luo ${ }^{1, a^{*}}$, Hu Cheng ${ }^{1, b}$, Jiong Wang ${ }^{1, c}$ \\ ${ }^{1}$ College of Mechatronic Engineering and Automation, National University of Defense Technology, \\ Changsha, Hunan, China \\ a407639648@qq.com, b13875851753@163.com, cwangjiong339@126.com
}

\begin{abstract}
Keywords: Permanent magnet synchronous motor, cascade linear active disturbance rejection controller, model compensation, load disturbance observer, robustness

Abstract. Permanent magnet synchronous motor (PMSM) has been widely used in industrial fields. The conventional control method for vector control of PMSM is PID, which has some disadvantages such as large overshoot, bad robustness. In this paper, a cascade control strategy with three first-order linear active disturbance rejection controllers( LADRC) is proposed, which can achieve not only cascade control of speed and q- axis current but also decoupling of d- axis and qaxis current. In order to improve the anti-disturbance ability, a load disturbance observer(LDO) is designed for model compensation. The simulation results show that, compared with PI controller, cascade LADRC method with model compensation has advantages of no overshoot, strong robustness and anti-disturbance ability.
\end{abstract}

\section{Introduction}

Owing to their characteristics of high power density, high efficiency and reliability, PMSMs are widely used in various industrial fields[1]. Vector control is one of the most commonly used control methods of PMSM, whose speed and current are generally regulated by proportional/integral/derivative (PID) controller. The conventional PID controller has the advantages of easy realization and simple control. However, it also has some disadvantages, such as large overshoot, long settling time and so on. It is difficult for the PMSM system to realize high precision control.

Therefore, in recent years, many methods are used to improve the conventional PID controller and enhance the control performance[2-6]. Active disturbance rejection controller(ADRC) proposed by Han is one of these methods which does not rely on the precise model, and has strong robustness and adaptability[7]. However, the tuning procedure of ADRC is very complicated due to its large number of parameters. To simplify the problem, the linear active disturbance rejection controller (LADRC) was proposed by Gao [8]. A second-order LADRC was proposed for the speed control, regarding current loop controller as part of the PMSM model[9]; LADRC was also introduced into sensorless vector control system of PMSM, tuning easily and showing good performance[10].

The aforementioned papers are mainly for the speed loop design. Regarding the speed loop and current loop as a whole, the use of cascade control can further improve the control performance of the system[11]. Based on this idea, three first-order LADRCs are designed for cascade control and decoupling in this paper. In addition, a LDO is designed for model compensation of speed LADRC. Simulation results show that the proposed controller can provide excellent control dynamic and static performance under large variations of model parameters and load disturbance compared with the conventional PI controller.

\section{The mathematical model of PMSM and LADRC}

The mathematical model of PMSM .On the $d q$ synchronous rotating frame of PMSM, the dynamic mathematical model of PMSM is shown as (1)-(3) [1] 


$$
\begin{aligned}
& \dot{\omega}_{r}=\frac{1}{J}\left(\frac{3}{2} n_{p} \psi_{f} i_{q}-B_{m} \omega_{r}-T_{l}\right) \\
& \dot{i}_{q}=\frac{1}{L_{q}}\left(u_{q}-R_{s} i_{q}-n_{p} \omega_{r} L_{d} i_{d}-n_{p} \omega_{r} \psi_{f}\right) \\
& \dot{i}_{d}=\frac{1}{L_{d}}\left(u_{d}-R_{s} i_{d}-n_{p} \omega_{r} L_{q} i_{q}\right)
\end{aligned}
$$

Where $J$ is moment of inertia, $B_{m}$ is friction coefficient, $\omega_{r}$ is rotor speed, $n_{p}$ is number of pole pairs, $T_{l}$ is load torque, $\psi_{f}$ is rotor magnetomotive force, $u_{d}$ is voltage of d-axis, $u_{q}$ is voltage of q-axis,$i_{d}$ is current of d-axis,$i_{q}$ is current of q-axis, $L_{d}$ is inductance of d-axis, $L_{q}$ is inductance of q-axis, $R_{s}$ is stator resistance.

The mathematical model of LADRC. For the sake of simplicity, consider a first-order plant

$$
\left\{\begin{array}{l}
\dot{x}_{1}=f(x, w(t), t)+b u \\
y=x_{1}
\end{array}\right.
$$

Where $y$ and $u$ are output and input, and $w(t)$ is external disturbance. Here, $f(x, w(t), t)$ refers to the total disturbance, including the unknown internal dynamics and the external disturbance $w(t)$. Define $f_{0}=f_{0}(\hat{x}, \hat{w}(t), t)$ as the knowable part of $f(x, w(t), t)$ and $f_{u}=f(x, w(t), t)-f_{0}(\hat{x}, \hat{w}(t), t)$ as the unknowable. Then the first-order LADRC can be designed as follows:

The Linear extended state observer (LESO) can be given as (5)

$$
\left\{\begin{array}{l}
\dot{z}_{1}=z_{2}-2 p_{0}\left(z_{1}-v_{1}\right)+b_{0} u+f_{0} \\
\dot{z}_{2}=-p_{0}^{2}\left(z_{1}-v_{1}\right)
\end{array}\right.
$$

Where $x_{2}=f_{u}$ is an augmented state, $p_{0}$ is the observer bandwidth, $f_{0}(\hat{x}, \hat{w}(t), t)$ is regarded as model compensation part. With the LESO designed, the controller is given by (6) and (7)

$$
\begin{aligned}
& u_{0}=k_{p}\left(v-z_{1}\right) \\
& u=u_{0}-\frac{z_{2}+f_{0}}{b}
\end{aligned}
$$

The framework of LADRC is shown in Fig.1.

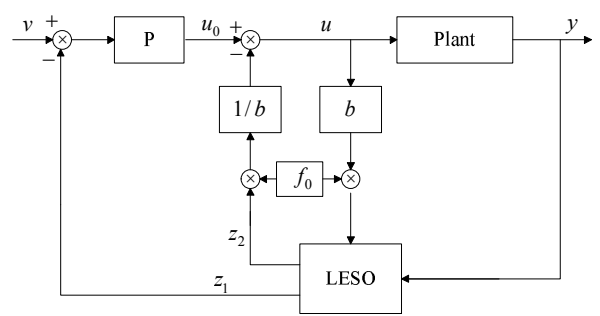

Fig.1 The block diagram of first-order LADRC

\section{The Controller Design}

Cascade LADRC for the Speed and q-Axis Current Loop. The mathematical models of the speed loop and q-axis current loop from (1) (2) are 


$$
\begin{aligned}
& \dot{\omega}_{r}=f_{1}+b_{1} i_{q} \\
& \dot{i}_{q}=f_{2}+b_{2} u_{q}
\end{aligned}
$$

Where $b_{1}=\frac{3}{2 J} n_{p} \psi_{f}, f_{1}=\frac{1}{J}\left(-B_{m} \omega_{r}-T_{l}\right), b_{2}=\frac{1}{L_{q}}$ and $f_{2}=\frac{1}{L_{q}}\left(-R_{s} i_{q}-n_{p} \omega_{r} L_{d} i_{d}-n_{p} \omega_{r} \psi_{f}\right)$. It can be seen that the "disturbance" $f_{2}$ contains the coupling terms, i.e., the product of $\psi_{f}$ and $\omega_{r}$, the product of $i_{d}$ and $\omega_{r}$. Since the given value of $i_{d}$ in vector control is zero, the latter is small. If the term of $i_{d}$ is omitted, the speed loop and q-axis current loop can be regarded as cascade system. Based on (8) and (9), the control structure of cascade LADRC can be designed as shown in Fig. 2.

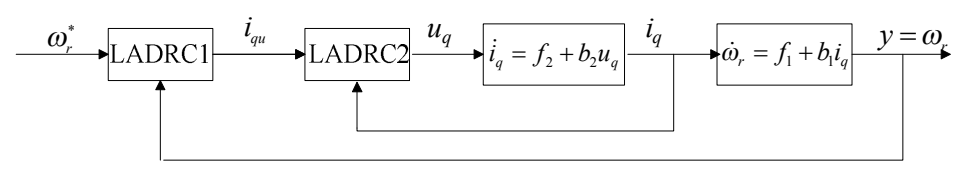

Fig.2 The control structure of cascade LADRC

From Fig.2, it can be seen that outer loop controller LADRC1 require $y$ to track $\omega_{r}^{*}$ and inner loop controller LADRC2 require $i_{q}$ to track $i_{q u}$. Then the actual control variables $u_{q}$ can be obtained.

To track the given input $\omega_{r}^{*}$ without overshoot, a function of the first-order tracking differentiator(TD) is given as (10) to arrange the ideal transient process.

$$
\dot{\omega}_{1}=-r f a l\left(\omega_{1}-\omega_{r}^{*}, a_{0}, \delta_{0}\right)
$$

Where the nonlinear function $f a l(e, \alpha, \delta)$ is given as $f a l(e, \alpha, \delta)=\left\{\begin{array}{l}|e|^{\alpha} \operatorname{sgn}(e),|e|>\delta \\ e / \delta^{1-\alpha},|e| \leq \delta\end{array}, a_{0}, \delta_{0}>0\right.$.

LADRC for d-Axis Current Loop.The model of the d-axis current loop from (3) is

$$
\dot{i}_{d}=f_{3}+b_{3} u_{d}
$$

Where $b_{3}=\frac{1}{L_{d}}$ and $f_{3}=\frac{1}{L_{d}}\left(-R_{s} i_{d}-n_{p} \omega_{r} L_{q} i_{q}\right)$. The "disturbance" $f_{3}$ contains the coupling terms, i.e., the product of $i_{q}$ and $\omega_{r}$.A first-order LADRC can also be designed for the decoupling of $i_{d}$ with $i_{q}$. The coupling term can be real-timely estimated and dynamically compensated by LESO of LADRC.

Load Disturbance Observer and Model Compensation. The term $f_{1}$ in (8) is the disturbance of the speed loop. It is difficult to ensure the estimation precision if $f_{1}$ is big. The load torque $T_{l}$ is the main factor that influences $f_{1}$. If $T_{l}$ could be observed and used for model compensation, the pressure of LESO for disturbance estimation reduces. Thus, a LDO is designed as follows.

From equation (1)

$$
\left[\begin{array}{c}
\dot{\omega}_{r} \\
\dot{T}_{l}
\end{array}\right]=\left[\begin{array}{cc}
-\frac{B_{m}}{J} & -\frac{1}{J} \\
0 & 0
\end{array}\right]\left[\begin{array}{l}
\omega_{r} \\
T_{l}
\end{array}\right]+\left[\begin{array}{c}
3 n_{p} \psi_{f} \\
2 J \\
0
\end{array}\right] i_{q}
$$

Choose $\boldsymbol{x}=\left[\begin{array}{ll}\omega_{r} & T_{l}\end{array}\right]^{T}$ as state variables, and $\boldsymbol{y}=\omega_{r}$ as output. The state and output equations are

$$
\left\{\begin{array}{l}
\dot{\boldsymbol{x}}=\boldsymbol{A x}+\boldsymbol{b} i_{q} \\
\boldsymbol{y}=\boldsymbol{C x}
\end{array}\right.
$$


Where $\boldsymbol{A}=\left[\begin{array}{cc}-\frac{B_{m}}{J} & -\frac{1}{J} \\ 0 & 0\end{array}\right], \boldsymbol{b}=\left(\begin{array}{c}\frac{3 n_{p} \psi_{f}}{2 J} \\ 0\end{array}\right), \boldsymbol{C}=\left[\begin{array}{ll}1 & 0\end{array}\right]$.

The observer is constructed as (14)

$\left\{\begin{array}{l}\dot{\hat{\boldsymbol{x}}}=\boldsymbol{A} \hat{\boldsymbol{x}}+\boldsymbol{b} i_{q}+\boldsymbol{K}(\boldsymbol{y}-\hat{\boldsymbol{y}}) \\ \hat{\boldsymbol{y}}=\boldsymbol{C} \hat{\boldsymbol{x}}\end{array}\right.$

Where $\hat{\boldsymbol{x}}=\left[\begin{array}{ll}\hat{\omega}_{r} & \hat{T}_{l}\end{array}\right]^{T}$ is estimated state vector, $\boldsymbol{K}=\left[\begin{array}{ll}k_{1} & k_{2}\end{array}\right]^{T}$ is the gain of the observer.

Define $\boldsymbol{e}=\boldsymbol{x}-\hat{\boldsymbol{x}}$ as the state error vector. The state error equation (15) is obtained by (13) and (14)

$\dot{\boldsymbol{e}}=\left[\begin{array}{cc}-\frac{B_{m}}{J}-k_{1} & -\frac{1}{J} \\ -k_{2} & 0\end{array}\right] \boldsymbol{e}$

If the desired poles of the observer are $\alpha$ and $\beta$, the characteristic equation can be obtained as

$$
\operatorname{det}(s \boldsymbol{I}-(\boldsymbol{A}-\boldsymbol{K} \boldsymbol{C}))=s^{2}+\left(\frac{B_{m}}{J}-k_{1}\right) s-\frac{k_{2}}{J}=s^{2}-(\alpha+\beta) s+\alpha \beta
$$

Then $\boldsymbol{K}$ is obtained as (17)

$$
\boldsymbol{K}=\left[\begin{array}{l}
k_{1} \\
k_{2}
\end{array}\right]=\left[\begin{array}{c}
-(\alpha+\beta)-B_{m} / J \\
-J(\alpha \beta)
\end{array}\right]
$$

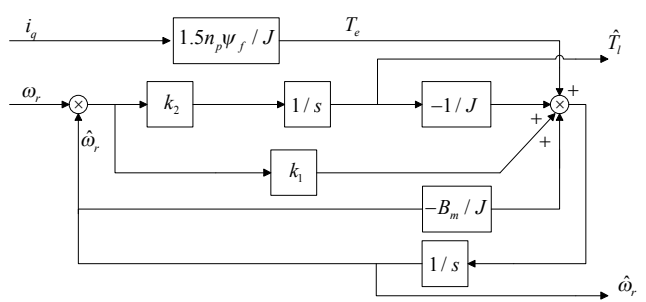

Fig.3. The block diagram of LDO

The LDO is designed as shown in Fig.3. When $\hat{T}_{l}$ is observed and $J$ is given, $f_{10}=\frac{1}{J}\left(-\hat{T}_{l}\right)$ can be regarded as the knowable part of $f_{1}$ and used for model compensation .

The Complete Control Algorithm. Based on the analysis above, the principle for cascade LADRC is shown in Fig. 4, and the complete algorithm of the controller is given as (18) and (19):

Controller for $\omega_{r}(\mathrm{LADRC} 1)$ and $i_{q}(\mathrm{LADRC})$ : 


$$
\left\{\begin{array}{l}
\dot{\omega}_{1}=-r_{1} f a l\left(\omega_{1}-\omega_{r}^{*}, a_{1}, \delta_{1}\right) \\
f_{10}=\frac{1}{J}\left(-\hat{T}_{l}\right) \\
\dot{z}_{11}=z_{12}-2 p_{1}\left(z_{11}-\omega_{1}\right)+b_{10} i_{q u}+f_{10} \\
\dot{z}_{12}=-p_{1}{ }^{2}\left(z_{11}-\omega_{1}\right) \\
u_{10}=K_{p 1}\left(\omega_{1}-z_{11}\right) \\
i_{q u}=u_{10}-\left(z_{12}+f_{10}\right) / b_{10} \\
\dot{z}_{21}=z_{22}-2 p_{2}\left(z_{21}-i_{q u}\right)+b_{20} u_{q} \\
\dot{z}_{22}=-p_{2}^{2}\left(z_{21}-i_{q u}\right) \\
u_{20}=K_{p 2}\left(i_{q u}-z_{21}\right) \\
u_{q}=u_{20}-z_{22} / b_{20}
\end{array}\right.
$$

Where $\omega_{1}$ is the transient process arranged for $\omega_{r}^{*} ; r_{1}, a_{1}$ and $\delta_{1}$ are parameters of the function $f a l$; $f_{10}$ is the model compensation part; $z_{11}, z_{21}$ track corresponding input of LESO $\omega_{1}, i_{q u}$ respectively; $z_{12}$, $z_{22}$ are the estimation of $f_{1}, f_{2} ; p_{1}, p_{2}$ are the observer bandwidth of LADRC1 and LADRC2 respectively; $K_{p 1}, K_{p 2}$ are the gains of $\mathrm{P}$ controller; $b_{10}, b_{20}$ are the estimation of $b_{1}, b_{2}$.

Controller for $i_{d}$ (LADRC3):

$$
\left\{\begin{array}{l}
\dot{z}_{31}=z_{32}-2 p_{3} z_{31}+b_{30} u_{d} \\
\dot{z}_{32}=-p_{3}{ }^{2} z_{31} \\
u_{30}=K_{p 3}\left(-z_{31}\right) \\
u_{d}=u_{30}-z_{32} / b_{30}
\end{array}\right.
$$

Where $z_{31}, z_{32}, p_{3}, K_{p 3}, b_{30}$ are defined similarly as corresponding variables of LADRC2.

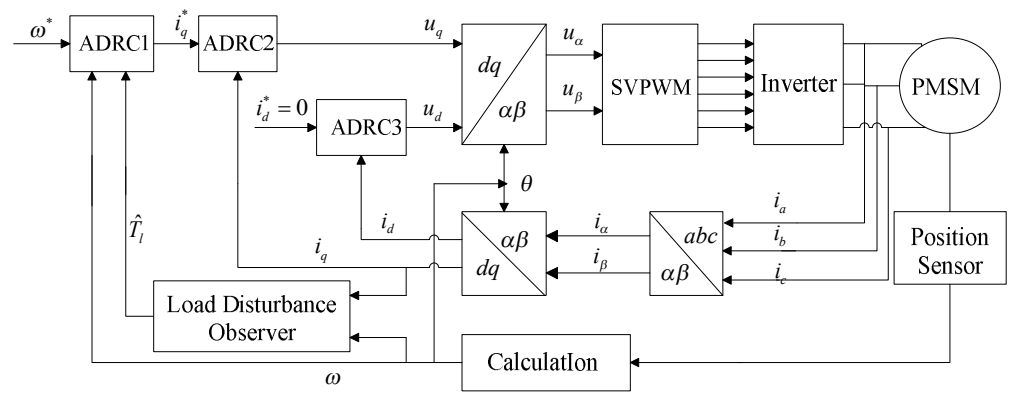

Fig.4. The block diagram of vector control system based on cascade LADRC

\section{Simulation Results}

To show the performance of the proposed controller, a MATLAB/Simulink model has been established . Each first-order ADRC is written by an S-function. Table 1 lists the parameters of PMSM and controller used in the simulation.

Table 1 The parameters of PMSM and controller

\begin{tabular}{|c|c|c|c|c|c|c|c|c|c|}
\hline \multicolumn{4}{|c|}{ PMSM Parameters } & \multicolumn{5}{c|}{ Controller Parameters } \\
\hline Name & value & $\begin{array}{c}\text { Nam } \\
\boldsymbol{e}\end{array}$ & value & Name & value & Name & value & Name & value \\
\hline$J$ & $6.2 \times 10^{-4} \mathrm{~kg} \cdot \mathrm{m}^{2}$ & $n_{p}$ & 4 & $r_{1}$ & 2000 & $K_{p 1}$ & 0.5 & $K_{p 2}$ & 10 \\
\hline$R_{s}$ & $0.18 \Omega$ & $\psi_{f}$ & $0.16667 \mathrm{~V} . \mathrm{s}$ & $a_{1}$ & 0.75 & $p_{2}$ & 8000 & $K_{p 3}$ & 10 \\
\hline$L_{d}$ & $0.835 \mathrm{mH}$ & $U_{N}$ & $120.92 \mathrm{~V}$ & $\delta_{1}$ & 0.1 & $p_{3}$ & 8000 & $\alpha$ & $-9 \times 10^{4}$ \\
\hline$L_{q}$ & $0.835 \mathrm{mH}$ & $T_{N}$ & $1 \mathrm{~N} . \mathrm{m}$ & $p_{1}$ & 1000 & $b_{20}$ & 1200 & $\beta$ & $-9 \times 10^{4}$ \\
\hline$B_{m}$ & $3 \times 10^{-4} \mathrm{~N} . \mathrm{m} . \mathrm{s}^{2} / \mathrm{rad}$ & & & $b_{10}$ & 1600 & $b_{30}$ & 1200 & & \\
\hline
\end{tabular}


The performance of the proposed controller is investigated under the following three cases :a) ideal condition; b) load disturbance and c) parameter variation. The proposed method is compared with the vector control based on the conventional PI controllers which are manually tuned to their desirable values.

Ideal Condition Performance. Fig. 5 shows the comparative simulation results of the step response of speed. The speed reference given is 500r/min. PI has an obvious overshoot which is nearly $10 \%$. Owing to the transient process arranged by the first-order TD, the proposed cascade LADRC has no overshoot and the shorter settling time.

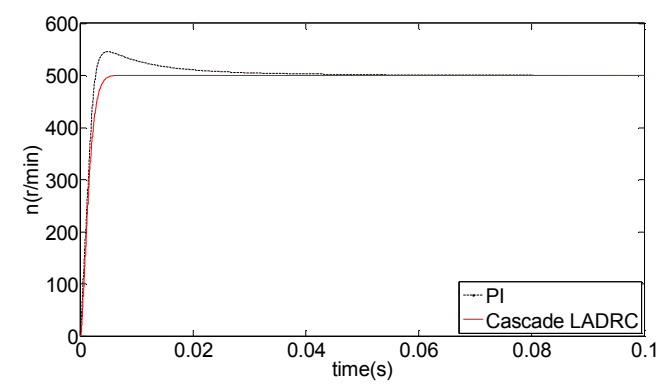

Fig.5. Comparative simulation result under ideal condition

Load Disturbance Performance. Fig. 6 shows the comparative simulation results when the load torque disturbance occurs, and steps up to $1 \mathrm{N.m}$ at $0.1 \mathrm{~s}$ and down to $0.7 \mathrm{~N} . \mathrm{m}$ at $0.13 \mathrm{~s}$. Fig. 6(a) shows the speed response curve. When the load torque changes, the proposed cascade LADRC can settle down to the speed reference given rapidly, whereas the PI has obvious speed drop or rise. This shows that the proposed controller has better performance compared with the PI controller from the viewpoint of anti-disturbance ability. Fig. 6(b) shows the load torque disturbance observed by LDO. The LDO can observe the applied load torque accurately and work well for the model compensation.

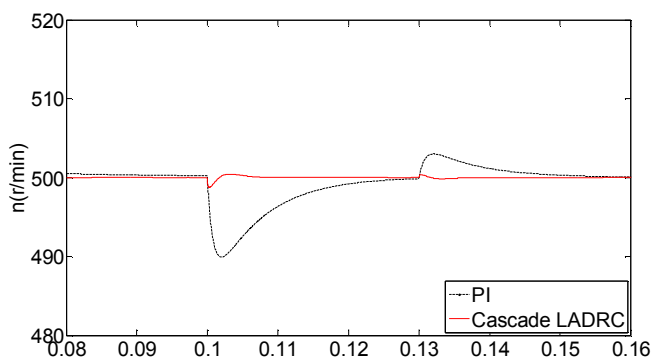

(a)

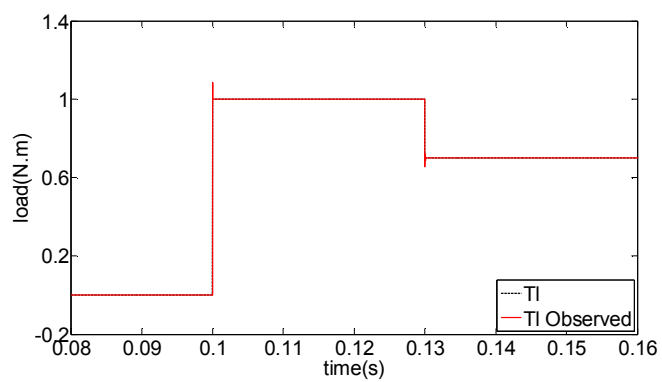

(b)

Fig. 6. (a) The speed response under load disturbances . (b) The load torque disturbance observed.

Parameters Variation Performance. Fig. 7 shows the comparative simulation results when the PMSM parameters vary. The motor parameters $J, \psi_{f}, L_{q}$ and $L_{d}$ change to $2.5,1.2,1.2,0.5$ times (becomes smaller when magnetic saturation occurs) of the original respectively. Load torque disturbance steps up to $1 \mathrm{~N} . \mathrm{m}$ at $0.1 \mathrm{~s}$ and down to $0.7 \mathrm{~N} . \mathrm{m}$ at $0.13 \mathrm{~s}$. Although, the parameters variation is not the general case, the proposed cascade LADRC can be evaluated under such rigid condition. Fig. 7(a) shows the speed response curve. The proposed cascade LADRC has nearly no overshoot and the shorter settling time than the PI controller. The strong robustness has been proved to the variation of the PMSM parameters. Fig. 7(b) shows the load torque disturbance observed. It can be seen that the disturbance observed is smaller than the applied value due to the variation of the PMSM parameters . The model compensation effect weakens when parameters vary greatly. 


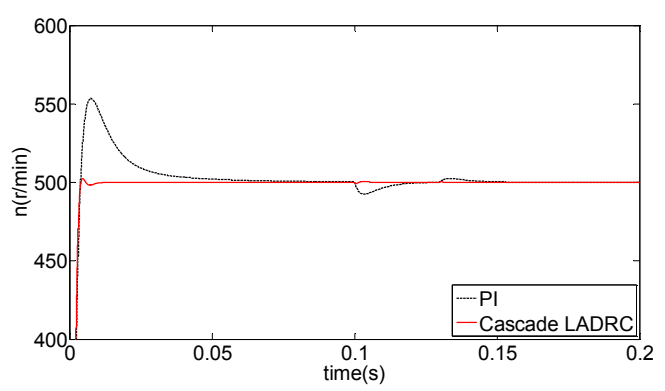

(a)

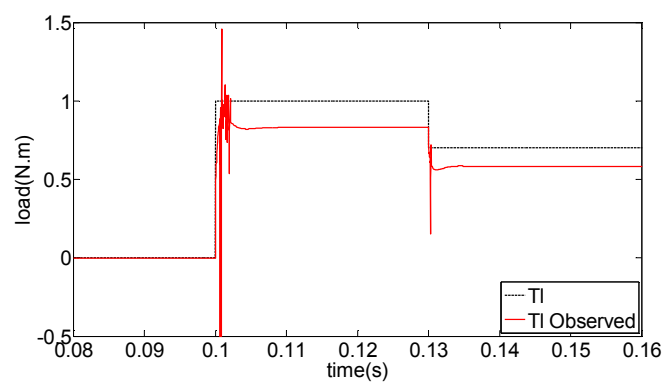

(b)

Fig. 7. (a) The speed response when parameters vary. (b) The load torque disturbance observed.

\section{Conclusions}

A novel cascade LADRC with model compensation has been presented in this paper for vector control of PMSM. The speed and q- axis current controllers are designed by two first-order LADRCs based on cascade control theory. The transient process is arranged in the speed LADRC resulting in fast response and no overshoot. Then the d- axis current LADRC is designed for the decoupling daxis and q-axis currents. A LDO is also designed for model compensation and further improvement of anti-disturbance ability. Simulation results show that the proposed method has the advantages of no overshoot, short settling time, good anti-disturbance ability and robustness to parameters variation when compared with the conventional PI controller.

\section{References}

[1] Renyuan Tang:Modern permanent magnet machines theory and design . ( Mechanical Industry Press, Beijing 2000).(in Chinese)

[2] P. Stewart and V. Kadirkamanathan: Dynamic Model Reference PI Control of Permanent Magnet AC Motor Drives, Control Engineering Practice, Vol. 9 (1998), p. 1255-1263.

[3] J. W. Jung, Y. S. Choi, V. Q. Leu, and H. H. Choi:Fuzzy PI-type current controllers for permanentmagnet synchronousmotors, IET Electr. Power App., Vol. 5 (2011), p. 143-152.

[4] V. M. Hernandez-Guzman and R. Silva-Ortigoza: PI control plus electric current loops for PM synchronous motors, IEEE Trans. Control Syst.Technol., Vol. 19(2011) ,p. 868-873.

[5] K. Y. Cheng and Y. Y. Tzou: Fuzzy optimization techniques applied to the design of a digital PMSM servo drive, IEEE Trans. Power Electron., Vol. 19(2004), p. 1085-1099.

[6] Wang G.J,Fong C.T,Chang K.J.:Neural-network-based self-tuning PI controller for precise motion control of PMAC motors.IEEE Trans. Ind. Electron., Vol.48(2001), p. 408-415.

[7] J. Han, Active disturbance rejection controller and its applications:Control and Decision, Vol. 13(1998), p.19-23. (In Chinese)

[8] Z. Gao: Scaling and bandwidth-parameterization based controller tuning,Proceedings of American Control Conference, 2003: 4989-4996.

[9] Zhigang Liu, Shihua Li: A second order ADRC algorith for PMSM . Proceedings of the 26th Chinese Control Conference, Vol.7(2007),p.68-71. (in Chinese)

[10] Jinliang Zhang,Longyun Kang:A sensorless vector control system of Permanent magnet synchronous motor based on line active disturbance rejection controller. $17^{\text {th }}$ ICEMS, (2014),p.1140-1144.

[11] Jinkun Liu: Advanced PID Control and Simulation (Electronic Industry Press, Beijing 2013). (in Chinese) 